\title{
EXPERIMENTAL MODEL OF BLADDER INSTABILITY IN RABBITS
}

\author{
K.T. BALASTEGHIN, A. M. NARDO, J.L. AMARO, C.R. PADOVANI \\ Departments of Urology and Statistics, Botucatu School of Medicine, UNESP, Botucatu, \\ São Paulo, Brazil
}

\begin{abstract}
Objective: Propose a new experimental model of bladder instability in rabbits after partial bladder obstruction.

Materials and Methods: Thirty North Folk male rabbits, weighting 1,700 to 2,820 g (mean: $2,162 \mathrm{~g}$ ) were studied. The animals were distributed in 2 experimental groups, formed by 15 rabbits each: Group 1 - clinical control. In this group there was no surgical intervention; Group 2 - bladder outlet obstruction. In this group, after anesthetizing the animal, urethral cannulation with Foley catheter $10 \mathrm{~F}$ was performed and then an adjustable plastic bracelet was passed around the bladder neck. It was then adjusted in order to not constrict the urethra. The following parameters were studied in M1 - pre-operative period; M2 - 4 weeks post-operatively moments: 1 )- urine culture; 2 )- cystometric study; 3)- serum creatinine and BUN.

Results: Bladder weight was 2.5 times larger in the group with obstruction than in the control group. Cystometric evaluation showed a significant increase in maximal vesical volume in the final moment at Group G2. However, there was no statistically significant difference among the groups studied. There was no statistically significant difference between maximal detrusor pressure and vesical compliance in the different moments or in the studied groups. There was an absence of uninhibited detrusor contractions in all the animals in group 1, and involuntary contractions were detected in 93\% of group 2 animals. There was no significant variation in BUN and serum creatinine either among the groups or in the same group.

Conclusions: We observed in the group with obstruction a bladder weight 2.5 higher than normal bladders. We detected involuntary contractions in $93 \%$ of the animals in group 2, establishing this experimental model as appropriate to secondary bladder instability and partial bladder outlet obstruction.
\end{abstract}

Key words: bladder, neurogenic; rabbits; urethral obstruction; urodynamics Int Braz J Urol. 2003; 29: 62-7

\section{INTRODUCTION}

Detrusor instability is a fair common phenomenon in the urological practice, occurring frequently in men after 50 years of age due to bladder outlet obstruction as a consequence of benign prostatic hyperplasia (1). In women it occurs in 30 to $50 \%$ of urinary incontinence cases (2). Despite the importance of this urinary dysfunction, pathophysiological alter- ations developing in the bladder due to the response to obstruction are not totally elucidated, and are difficult to investigate in humans. Thus, there are different experimental models described for partial bladder outlet obstruction using guinea pig (3), rabbit $(4,5)$, cat (6), dog (7), pig (8), and rat (9). The models described aimed to characterize bladder functional alterations caused by partial obstruction, but few works take into account the development of uninhib- 
ited detrusor contractions (9-13). This obstruction was performed in most cases constricting the urethrovesical junction with a suture tie $(14,15)$, a polyethylene tube (16), or a silicone strip (17). Due to the difficulty in reproducing these experiments, we propose a new experimental model of bladder instability in rabbits.

\section{MATERIALS AND METHODS}

Thirty male North Folk rabbits, weighting from $1,700 \mathrm{~g}$ and $2,820 \mathrm{~g}$ (mean 2,140 \pm 200 ) were used.

The animals were distributed in 2 experimental groups, formed by 15 rabbits each, separated as follows: a)- Group 1 (clinical control): In this group there was no surgical intervention, the animals were used as clinical and cystometric parameters of normality; b)- Group 2: a bladder outlet obstruction was performed. The animals received a pre-anesthetic solution consisting in diazepam $0.1 \%$ by intravenous route (diazepam $10 \mathrm{mg}+8 \mathrm{~mL} \mathrm{NaCl} 0.9 \%$ ) in $1 \mathrm{mg} /$ $\mathrm{kg}$ doses. Then they were anesthetized with Rompum and Ketalar, equal parts, $0.25 \mathrm{mg} / \mathrm{kg}$ by intramuscular route. Trichotomy and antisepsis with povidone in abdominal area, and cavity opening by distal median laparotomy from $2.0 \mathrm{~cm}$ of the pubis identifying the bladder and dissecting the ureters (Figure-1) were performed. Then, an adjustable polyethylene bracelet (Figure-2) was passed around the bladder neck

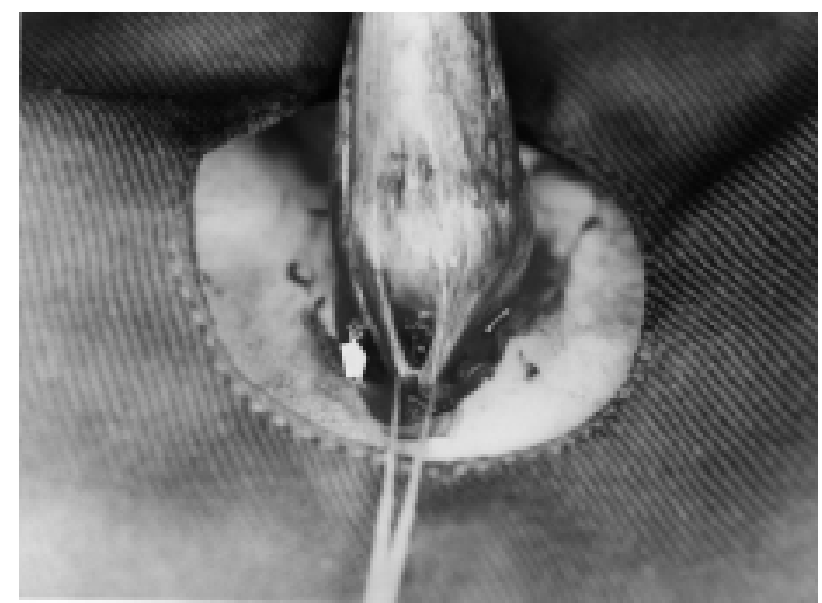

Figure 1 - Demonstration of the ureteral position after dissection (arrow).

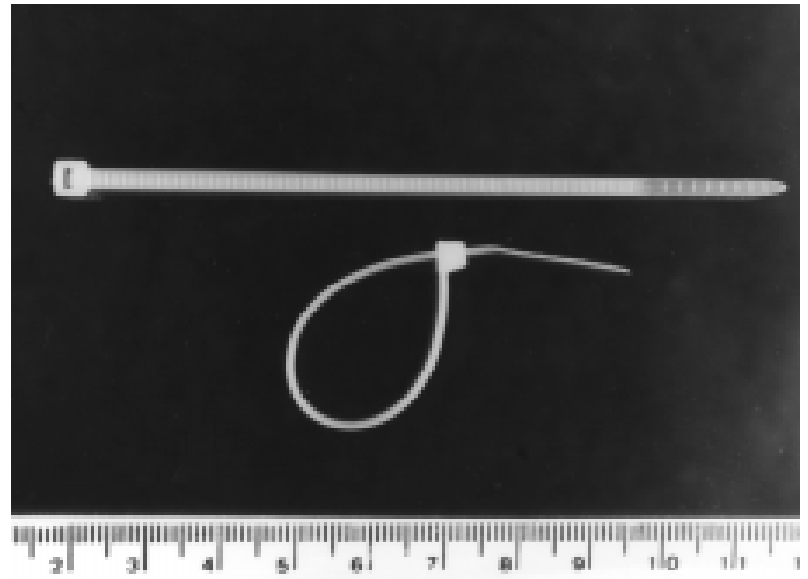

Figure 2 - Detail of the polyethylene adjustable bracelet.

(Figure-3), being then adjusted in order to not constrict the urethra which was previously catheterized with a Foley $10 \mathrm{~F}$ catheter. The following exams were performed in all animals in different moments - initial (M1) and after 4 weeks (M2) - : urine culture, serum creatinine and BUN, and cystometric study. Animals presenting positive urine culture were treated with Trimethoprim-Sulfamethoxazole, $1 \mathrm{mg} / \mathrm{kg} /$ day by intramuscular route.

The cystometric study was performed using Urobyte $^{\mathrm{TM}} 5000$ computed urodynamics system. To measure intravesical pressure a $10 \mathrm{~F}$ Foley catheter was used. To measure intra-abdominal pressure, a catheter with rectal balloon described by Amaro et al. (18) was used. During this exam the vigil animals were placed in wooden goiters, with posterior extremities held in cotton string. After antisepsis of the penile area with topic Povidone and lubrication with $2 \%$ Xylocaine $^{\mathrm{TM}}$ jelly, a 10F double-way Foley catheter was introduced into the bladder with vesical emptying, and urine was drawn in a sterile tube for urine culture.

After lubricating the rectal balloon with $2 \%$ Xylocaine ${ }^{\mathrm{TM}}$ jelly, it was introduced $2 \mathrm{~cm}$ beyond the anal margin and connected to a 2-way tap, injecting $0.4 \mathrm{~mL}$ sterile water in one of the ways in order that the balloon wall and the rectal mucosa came into contact. The other way was connected to the pressure transmitter.

The vesical catheter was connected to a 2way tap, and one of the ways was connected to the 

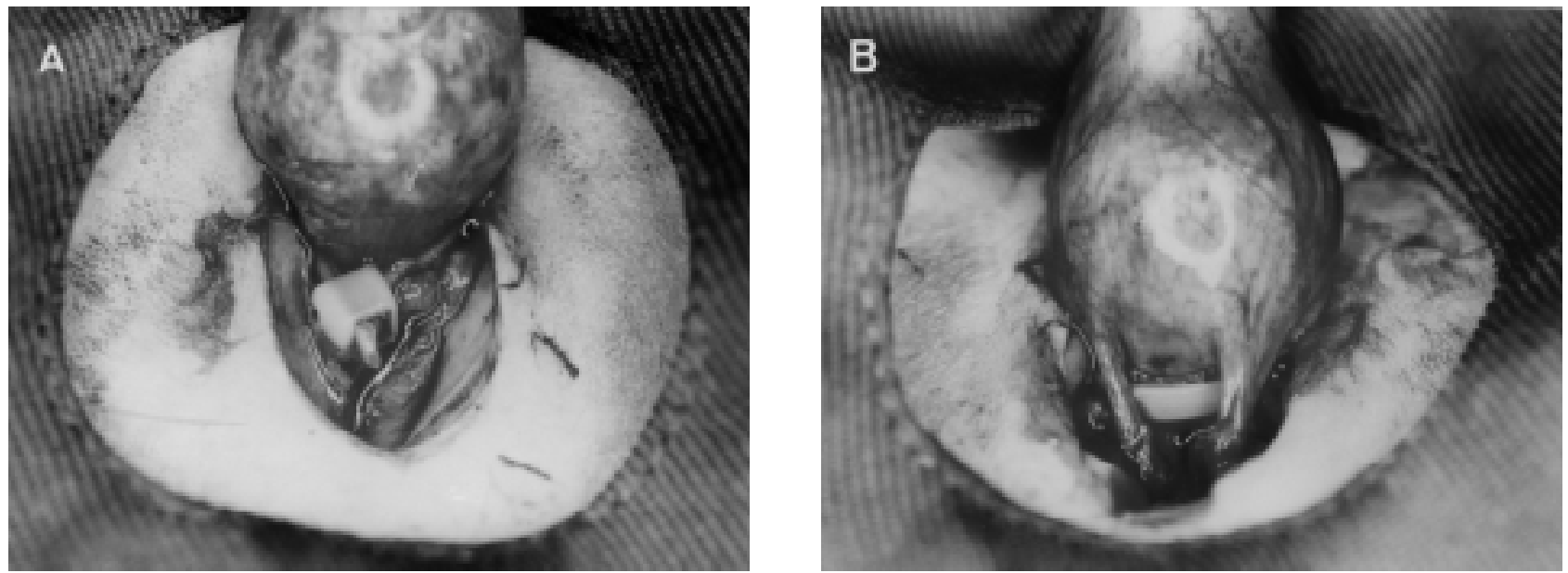

Figure 3 - Schematic illustration of bladder neck area where the polyethylene adjustable bracelet was placed, showing the ureters intact. A) Anterior view and B) Posterior view.

pressure transmitter, and the other to a continuous infusion pump ( $2 \mathrm{~mL} / \mathrm{min})$. To verify the good placement of the catheters, a slight compression of the abdomen was made in order to obtain an abdominal pressure curve, that was transmitted to the bladder and, consequently, also obtaining the vesical pressure curve.

The cystometry was initiated proceeding to the simultaneous measurements of the abdominal and vesical pressures. When urinary leakage around the vesical catheter was observed, the exam was discontinued. In this moment we verified the vesical pressure (VP) and the abdominal pressure, also measured in the beginning of the urinary leakage $(\mathrm{VP}-\mathrm{AbdP})$ expressing maximal detrusor pressure (DetP). Maximal bladder capacity was considered the one which attained a filling volume enough to yield bladder contractions. Vesical compliance was obtained by the formula: $\mathrm{Fv}-\mathrm{Iv} / \mathrm{Fp}-\mathrm{Ip}$ where $\mathrm{Fv}=$ Final Volume, Iv $=$ Initial volume, $\mathrm{Fp}=$ Final Pressure, $\mathrm{I} p=$ Initial Pressure.

We considered uninhibited contractions those detrusor involuntary contractions with low vesical volume, yielding or not simultaneous urinary leakage (Figure-4).

The study of the combination of urine culture and uninhibited contractions in the beginning and the end of the evaluation, in the different groups, was performed through Mac Nemar test. Comparison of the groups' mean profile along both moments of evaluation was performed through the analysis of repeated measures, considering both groups independently. All comparisons were performed with $5 \%$ level of significance.

\section{RESULTS}

There was a significant increase in bladder weigh in G1 compared to G2. After 4 weeks mean

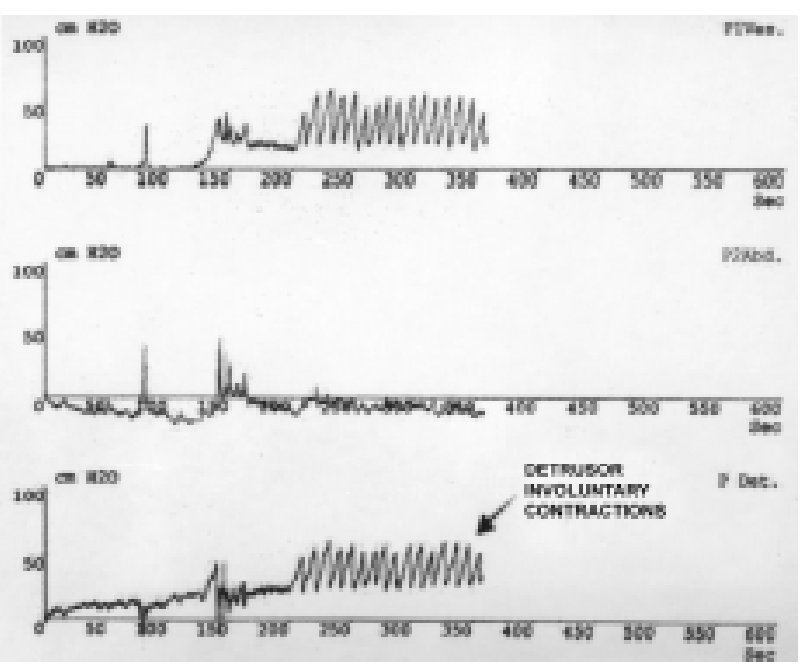

Figure 4 - Diagram showing detrusor involuntary contractions in an animal from group $G 2$. 
Table $1-$ Mean \pm standard deviation of maximal vesical volume in rabbits of the experimental groups.

\begin{tabular}{lcc}
\hline Group & \multicolumn{2}{c}{ Evaluation Moment } \\
& Initial (mL) & Final (mL) \\
\hline G1 & $36.13 \pm 24.78$ & $42.33 \pm 21.01$ \\
G2 & $35.13 \pm 13.60$ & $54.53 \pm 22.77$ \\
\hline
\end{tabular}

bladder weight in the G2 animals (bladder outlet obstruction) was 2.5 higher than those in the clinical control group (G1). In cystometric evaluation we observed that in absolute figures there was a trend toward greater bladder capacity in the final moment for group G1 (Table-1), however, there was no statistically significant difference. In group G2, maximal bladder volume $\left(\mathrm{V}_{\text {max }}\right)$ was significantly higher in the final moment compared to initial moment (Table-1). There was no statistically significant difference between the groups in the study.

In the analysis of maximal detrusor pressure there was no statistically significant difference between the groups, and in the different moments (M1 and M2) of the maximal detrusor pressure (Table-2).

We have observed no statistically significant difference in bladder compliance between the groups in different moments (M1 and M2) (Table-3).

In the assessment of the uninhibited detrusor contractions there was absence of contractions in all the animals in group 1 (control), and uninhibited contractions were detected for $93 \%$ in group 2 .

There was no statistically significant difference between the groups or for different moments studied for BUN analysis.

In the initial moment, mean plasma creatinine for G1 was inferior to G2, however there was no

Table 2 - Mean \pm standard deviation of detrusor pressure in cm of water for rabbits in the experimental groups.

\begin{tabular}{|c|c|c|}
\hline \multirow[t]{2}{*}{ Group } & \multicolumn{2}{|c|}{ Evaluation Moment } \\
\hline & Initial $\left(\mathrm{mL} / \mathrm{cm} \mathrm{H}_{2} \mathrm{O}\right)$ & Final $\left(\mathrm{mL} / \mathrm{cm} \mathrm{H}_{2} \mathrm{O}\right)$ \\
\hline G1 & $36.47 \pm 15.03$ & $27.27 \pm 13.47$ \\
\hline $\mathrm{G} 2$ & $32.60 \pm 14.47$ & $31.40 \pm 15.42$ \\
\hline
\end{tabular}

Table 3 - Mean \pm standard deviation of vesical compliance in $\mathrm{mL} / \mathrm{cm}$ of water for rabbits in the experimental groups.

\begin{tabular}{lcr}
\hline \multirow{2}{*}{ Group } & \multicolumn{2}{c}{ Evaluation Moment } \\
& Initial $\left(\mathbf{m L} / \mathbf{c m ~ H}_{\mathbf{2}} \mathbf{O}\right)$ & \multicolumn{1}{c}{ Final $\left(\mathbf{m L} / \mathbf{c m ~ \mathbf { H } _ { 2 } \mathbf { O } )}\right.$} \\
\hline $\mathrm{G} 1$ & $2.79 \pm 1.47 \mathrm{Aa}$ & $3.40 \pm 1.71 \mathrm{Aa}$ \\
$\mathrm{G} 2$ & $2.81 \pm 2.10 \mathrm{Aa}$ & $2.83 \pm 1.67 \mathrm{Aa}$ \\
\hline
\end{tabular}

statistically significant difference. Indeed, there was a statistically significant difference between groups G1 and G2 in the final moment.

In urine culture analysis for the animals studied, at moment M1, we have detected a positive culture for $40 \%$ of the cases. There was a higher predominance of Pseudomonas aeruginosa followed by Echerichia coli. At moment M2 we found positive culture for $50 \%$ of the cases, with predominance of Escherichia coli, followed by Pseudomonas aeruginosa, and Klebsiella pneumoniae. We did not observe a correlation between culture and the presence of uninhibited detrusor contractions.

\section{DISCUSSION}

The increase of bladder weight is a major alteration in all models of bladder obstruction (19). We have observed that after 4 weeks of partial obstruction, bladder weigh in the animals of $\mathrm{G} 2$ group (obstructed) was 2.5 times higher than in G1 group (control). Other authors $(13,15,17)$ observed a significant increase in rabbits' bladder weight after 4 weeks of partial obstruction. This fact could occur due to the presence of hypertrophy and hyperplasia of vesical wall (20).

Different studies with partial bladder obstruction are reported in literature. However, initially the reproduction of these models was not possible, for in some of them we observed the displacement of the polyethylene catheter or the silicone strip, yielding failure of the bladder obstruction and the non-development of uninhibited detrusor contractions. Contrarily, when sutures were performed at the extremities of these catheters to avoid this mobilization in many cases we observed an excessive obstruction, 
promoting bladder lithiasis or animal death. This fact, tends to demonstrate the difficulty of reproducing the models reported in literature $(16,17)$. We observed that the use of a polyethylene adjustable bracelet allowed not only standardization of the degree of obstruction, but still the development of uninhibited detrusor contractions in $93 \%$ of the cases.

Regarding BUN we did not find statistical difference between the groups in the different moments showing that partial obstruction of the bladder did not influence urea metabolism in the animals studied.

The analysis of plasma creatinine did not show statistically significant difference between control (G1) and obstructed (G2) groups at the initial moment. However, at the final moment we did find statistically significant difference between the groups. This may be explained by the presence of bladder instability in G2 group. However, although this statistically significant difference was observed, there was no positive correlation at the initial moment or in different moments in G2 group, and this may relate to a biologic or methodological variation.

\section{CONCLUSION}

The present study demonstrates that the experimental model is appropriate to study secondary bladder instability due to partial bladder obstruction.

Support: FAPESP

\section{REFERENCES}

1. Andersson KE: Current concepts in the treatment of disorders of micturition. Drugs. 1988; 35: 477- 94.

2. Wise BG, Cardozo LD: The urge syndrome. Curr Obstet Gynecol. 1992; 2: 105-10.

3. Mostwin JL, Karim OMA, Van Koeveringe G, Brooks EL: The guinea pig as a model of gradual urethral obstruction. J Urol. 1991; 145: 854-8.

4. Brent L, Stephens FD: The response of smooth muscle cells in the rabbit urinary bladder to outflow obstruction. Invest Urol. 1975; 12: 494-502.
5. Mayo ME, Hinman F: Structure and function of the rabbit bladder altered by chronic obstruction or cystitis. Invest Urol. 1976; 14: 6-9.

6. Levin RL, Longhurst PA, Barasha B, Mcguire EJ, Elbadawi A, Wein AJ: Studies on experimental bladder outlet obstruction in the cat. Long term functional effects. J Urol. 1992; 148: 939-43.

7. Broderick GA, Longhurst PA, Juniewicz PE, Wein AJ, Levin RL: A novel canine model of parcial outlet obstruction secondary to prostatic hypertrophy. World J Urol. 1994; 12: 245-8.

8. Sibley GNA: The Psychological response of the detrusor muscle to experimental bladder out flow obstruction in the pig. Br J Urol. 1987; 60: 332-6.

9. Lluel P, Duquenne C, Martin D: Experimental bladder instability following bladder outlet obstruction in the female rat. J Urol. 1998; 160: 2253-7.

10. Sibley GNA: An experimental model of detrusor instability in the obstructed pig. Br J Urol. 1985; 57: 292-8.

11. Malmgreen A, Sjogreen C, Uvelius B, Mattiasson A, Andersson K-E, Andersson PO: Cystometrical evaluation of bladder instability in rats whit infravesical outflow obstruction. J Urol. 1987; 137: 1291-4.

12. Gray M: Progressive changes in detrusor function with bladder outlet obstruction. J Urol. 1997; 158: 1-3.

13. Kwak TI, Lee JG: Inhibitoty effects of propiverine, atropine and oxybutinin on bladder instability in rats with infravesical outlet obstruction. Br J Urol. 1998; 82: 272-7.

14. Levin RM, High J, Wein AJ: The effect of short-term obstruction on urinary bladder function in the rabbit. $\mathrm{J}$ Urol. 1984; 132: 789-91.

15. Schroder A, Chichester P, Barry A, Penelope K, Longhurst A, Lieb J, et al.: Effect of chronic bladder outlet obstruction on blood flow of the rabbit bladder. J Urol. 2001; 165: 640-6.

16. Ghoniem GM, Regnier CH, Biancani P, Johnson L, Susset JG: Effect of vesical outlet obstruction on detrusor contractility and passive properties in rabbits. $\mathrm{J}$ Urol. 1986; 135: 1284-9.

17. Kato K, Wein A, Kitada S, Haugaard N, Levin RM: The functional effect of mild outlet obstruction on the rabbit urinary bladder. J Urol. 1988; 140: 880-4.

18. Amaro JL, Cury PR, Fabris VH, Trindade JCS: Ampliação vesical utilizando dura-máter e pericárdio bovino. Estudo comparativo em coelhos. J Bras Urol. 1997; 23: 88-92.

19. Nigro DA, Haugaard N, Wein AJ, Levin RM: Metabolic basis for contractile dysfunction following 


\title{
BLADDER INSTABILITY IN RABBITS
}

chronic parcial bladder outlet obstruction in rabbits. Mol Biol Biochem. 1999; 200: 1-6.

20. Pampinela F, Roelofs M, Castelucci E, Passerine-
Glazel G, Pagano F, Sartore S: Time-dependent remodeling of the bladder wall in growing rabbits after parcial outlet obstruction. J Urol. 1997; 157: 677-82.

Received: June 26, 2002

Accepted after revision: December 12, 2002

\author{
Correspondence address: \\ Dr. Karina Tuma Balasteghin \\ Departamento de Urologia \\ Faculdade de Medicina de Botucatu - UNESP \\ Distrito de Rubião Júnior, s/n. \\ Botucatu, SP, 18618-970, Brazil \\ Fax: + 5514 6802-6271 \\ E-mail: karinatuma@uol.com.br
}

